\title{
Evaluation of Traumatic Knee Joint Injuries with MRI
}

\author{
Rajesh Umap ${ }^{1}$, Bijpuriya Anurag ${ }^{2}$, Sachin Bagale ${ }^{3}$, Navid Shattari ${ }^{4}$ \\ ${ }^{1}$ Associate Professor, ${ }^{2}$ Senior Resident, ${ }^{3}$ Associate Professor, ${ }^{4}$ Senior Resident, Department of Radiodiagnosis, BJGMC Pune, \\ Maharashtra, India
}

Corresponding author: R. A. Umap, E/604, Apostrophe Society, Opposite NCP Office, Wakad - Thergaon Link Road, Wakad, Pune, Maharashtra, India

DOI: 10.21276/ijcmsr.2018.3.3.17

【

How to cite this article: Rajesh Umap, Bijpuriya Anurag, Sachin Bagale, Navid Shattari. Evaluation of traumatic knee joint injuries with MRI. International Journal of Contemporary Medicine Surgery and Radiology. 2018;3(3):C77-C81.

\section{A B S T R A C T}

Introduction: The knee joint is a biggest joint of the human body with complex articulation characterized by the presence of ligamentous and meniscal structures that play an important role in the stability and mobility. MRI due to its excellent soft tissue contrast resolution and multiplanar imaging capabilities provides significant advantages over other imaging techniques in the evaluation of traumatic injuries of knee joint. Study aimed to study the role of MRI in the evaluation of traumatic injuries of knee joint.

Material and methods: A total number of 100 patients referred with history of knee injury were imaged with 1.5 Tesla GEsigna HdxII MRI machine in the department of radiology over a period of 18 months.

Results: Commonest injuries detected in the study are anterior cruciate ligament tear, tear of posterior horn of medial meniscus, bone contusions and joint effusions. Clinical presentation and radiographs of the patient did not help in diagnosis in most of the cases of acute knee injury, especially in multiple ligament and bone injuries. MRI detected soft tissue injuries very well in addition to the bony injuries.

Conclusion: Magnetic resonance imaging is the excellent non invasive investigation tool for knee injury due to excellent soft tissue contrast resolution and multiplanar imaging capabilities which provides the most detailed evaluation in cases of various soft tissue injuries of knee joint.

Key Words: Traumatic Knee, Joint Injuries, MRI

\section{INTRODUCTION}

The knee joint is a biggest joint of the human body with complex articulation characterized by the presence of ligamentous and meniscal structures that play an important role in the stability and mobility ${ }^{1}$. This articulation is subject of very high mechanical stresses. The frequency, diversity and severity of ligament and meniscus injuries occur especially in the young and sportsmen, associated with significant morbidity, frequently need surgical treatment and extensive rest. Joint injury has been recognized as a potent risk factor for the onset of osteoarthritis ${ }^{2}$. Although clinical examination is important for the diagnosis of ligament and meniscal injury, painful stress examinations are not always accurate in the acute phase of the injury. In cases of knee joint trauma, clinical examination along with radiographs and even CT scan is not enough to diagnose many internal derangements of this joint. MRI, due to its excellent soft tissue contrast resolution and multiplanar imaging capabilities provides significant advantages over other imaging techniques in the evaluation of traumatic injuries of knee joint ${ }^{3}$. MRI has revolutionized diagnostic imaging of the knee. It provides excellent soft tissue contrast and is capable of evaluating the soft tissue and bony structures in multiple imaging planes which provide significant advantages over other imaging techniques. It represents a non-invasive and radiation-free technique that provides access to a real "lesional mapping". It plays a crucial role in the diagnosis and monitoring of traumatic knee injuries. MRI has also been demonstrated as a cost effective technique by reducing unnecessary surgical and arthroscopic interventions ${ }^{4}$.

Current research aimed to study the normal anatomy of knee joint on MRI, to study efficacy of MRI in the evaluation of traumatic injuries of knee joint, to study MRI presentations of various traumatic injuries of knee joint and to study the correlation between clinical presentations and radiological findings.

\section{MATERIAL AND METHODS}

A total number of 100 patients referred with history of knee injury were imaged with 1.5 Tesla GE-signa HdxII MRI machine in the department of radiology over a period of 18 months.

It was a observational study and a total of 100 patients fulfilling the selection criteria were studied.

Source of data:- Patients referred from outpatient department of tertiary care centre with history of knee injury. 


\section{Selection criteria}

Inclusion criterion: Patients referred with history of knee injury.

\section{Exclusion criteria}

1. All patients who did not give consent to be a part of the study.

2. Patients with ferromagnetic implants, pacemakers, cochlear implants and aneurismal clips.

3. Degenerative arthritis, infection, neoplasm and any previous surgery to the knee.

The ethical clearance was obtained from Ethics Committee of B.J. Government Medical College, Pune. Patients fulfilling the selection criteria were informed about the purpose and nature of the study and were enrolled after obtaining a written informed consent.

The examination was done using 1.5 Tesla GE-signa HdxII MRI machine. Super conductive magnet with a useful aperture of $60 \mathrm{~cm}$ in diameter and dedicated eight-channel knee coil. The overall length of the system was $160 \mathrm{~cm}$. The imaging system is enclosed in a radio frequency room.

\section{Recommended sequences}

Statistical analysis:- The data obtained was coded and entered into Microsoft Excel Worksheet (Annexure III). The categorical data was expressed as rates, ratios, proportions and percentages.

\section{RESULTS}

This was a prospective study of 18 months duration, done at department of Radiodiagnosis, B.J. government medical college, Pune aiming at evaluating the role of MRI in all types of knee injuries. A total of 100 patients with history of injury to knee joint referred for MRI scan of knee joint were studied. Majority of the patients were male (80\%) and (20\%) patients were female with the male to female ratio is $4: 1$. The commonest age group was 21 to 30 years for both males and females with mean age of 31.67 years for male and 35.1 years

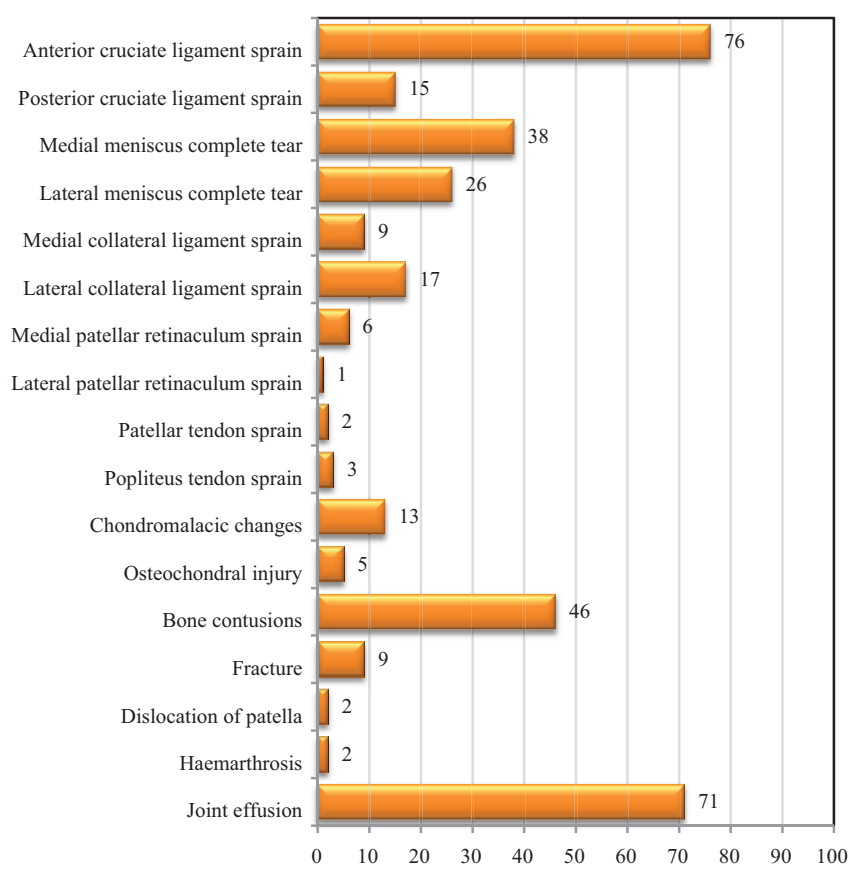

Graph-1: Various findings in 100 patients. for female.

Clinical presentation of the patient did not help in diagnosis in most of the cases of acute knee injury, especially in multiple ligaments injuries. Among the knee injuries, the most commonly injured structure was anterior cruciate ligament (76\%), followed by medial meniscus (38\%), lateral meniscus (26\%), lateral collateral ligament (17\%), posterior cruciate ligament(15\%) and medial collateral ligament (9\%), medial patellar retinaculum (6\%), lateral patellar retinaculum (1\%), patellar tendon (2\%) and popliteus tendon (2\%) (graph-1). Out of total 76 ACL injuries, low grade partial ACL tear was commonest (47.4\%), followed by complete tear (25\%), high grade partial tear $(21.0 \%)$ and grade I sprain $(6.6 \%)$. The most common location of complete tear was midsubstance (47.4\%), followed by femoral attachment (42.1\%) and tibial attachment (10.5\%). Commonest location of tear in menisci was posterior horn of medial meniscus (46.2\%) followed by
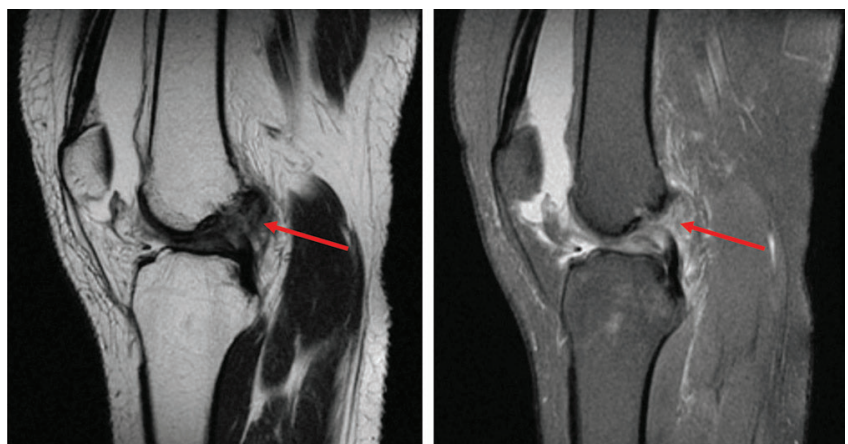

Figure 1 and 2: SAG T2W and SAG PDFS images showing complete ACL tear (Red arrows)
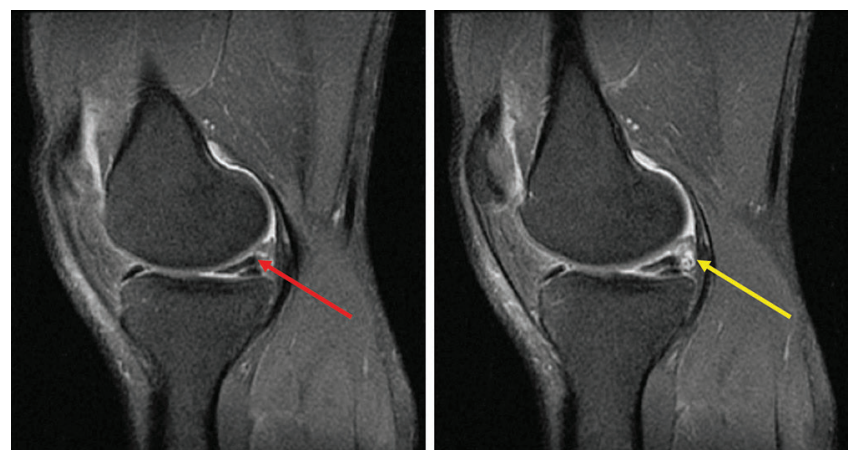

Figure 3 and 4: SAG PDFS images showing horizontal tear of anterior horn of medial meniscus (Red arrow) with parameniscal cysts (Yellow arrow).
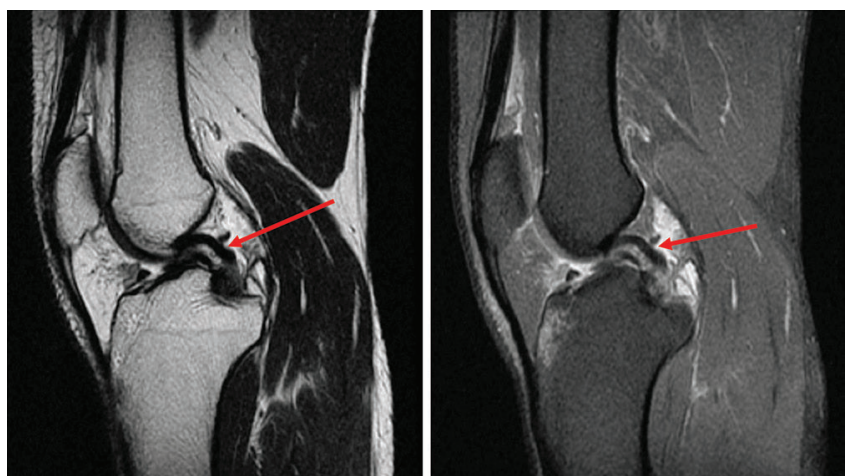

Figure 5 and 6: SAG T2W and SAG PDFS image showing bucket handle tear of medial meniscus with double PCL sign (Red arrows). 


\begin{tabular}{|l|l|c|c|c|c|c|}
\hline Sequence & Fat Saturation & FOV $(\mathbf{c m})$ & Matrix & TR (ms) & TE (ms) & Slice Thickness / Gap (mm) \\
\hline COR T1 & No & 16 & $256 \times 224$ & 1000 & 7.4 & $3 / 0$ \\
\hline AX T1 & No & 16 & $256 \times 224$ & 1000 & 7.4 & $3 / 0$ \\
\hline SAG T2 FS & No & 16 & $256 \times 224$ & 5140 & 88 & $3 / 0$ \\
\hline COR T2 FS & No & 16 & $256 \times 224$ & 5460 & 72 & $3 / 0$ \\
\hline COR STIR & Yes & 16 & $224 \times 160$ & 3000 & 52 & $3 / 0$ \\
\hline Intermediate PD AX & Yes & 16 & $224 \times 192$ & 4480 & 26 & $3 / 0$ \\
\hline Intermediate PD SAG & Yes & 16 & $224 \times 192$ & 4480 & 26 & $3 / 0$ \\
\hline Intermediate PD COR & Yes & 16 & $224 \times 192$ & 4480 & 26 & $3 / 0$ \\
\hline SAG 3D GRE & Yes & $256 \times 160$ & 12 & 5 & $3 / 0$ \\
\hline \multicolumn{7}{|l|}{ Table-1: Routine Mr imaging protocol: knee (volume surface phased array) coil } \\
\hline
\end{tabular}

posterior horn of lateral meniscus (16.7\%), anterior horn of lateral meniscus (14.1\%), body of medial meniscus (12.8\%), body of lateral meniscus (7.7\%) and anterior horn of medial meniscus (2.5\%). Some of the tears located in one part were noted extending to other parts of meniscus. Horizontal tear was commonest (12\%) followed by complex tear (11\%), bucket handle tear (07\%), radial tear (3\%) and longitudinal tear $(2 \%)$ in medial meniscus. Commonest type of tear in lateral meniscus was horizontal tear (42.3\%) followed by complex tear (26.9\%), longitudinal tear (15.5\%), vertical radial tear (11.5\%) and oblique tear (3.8\%). MCL injury was associated with ACL and medial meniscus injuries. Similarly LCL tear showed a relationship with lateral meniscus tear (figure 1-6).

Patellar dislocation was noted in two patients. One patient with patellar dislocation was found to be having medial patellar retinaculum sprain also. $42.1 \%, 47.36 \%, 36.84 \%$ and $37.5 \%$ of ACL, PCL, medial meniscus and lateral meniscus injuries were associated with bone contusions. Injuries of PCL were noted commonly associated with bone contusions. From the present study, it was noted that Magnetic Resonance Imaging is most comprehensive, non invasive and safe modality in radiological evaluation of knee injury.

\section{DISCUSSION}

The knee is an anatomically and biomechanically complex joint. The single most common indication of performing a knee MRI is to diagnose internal derangements in an injured knee.

Out of total 100 patients, major cause of knee joint trauma was fall (32\%), followed by road traffic accident (31\%), sports injury (27\%) and twisting injury (10\%).

Pain (100\%), followed by instability (42\%), joint stiffness/ abnormal locking/decreased range of motion (27\%) and swelling (7\%) are the common presenting complaints of knee joint trauma. In the present study, there was wide variation in the MRI diagnosis and patient presented with clinical complaints. The clinical diagnosis of anterior cruciate ligament injury was noted in $42 \%$ of the study population, followed by meniscal injury (27\%). The MRI findings noted were anterior cruciate ligament injury (76\%), followed by medial meniscal injury (38\%), lateral meniscus (26\%), lateral collateral ligament (17\%), posterior cruciate ligament (15\%), medial collateral ligament (9\%), medial patellar retinaculum (6\%), lateral patellar retinaculum (1\%), patellar tendon (2\%) and popliteus tendon (2\%). We found clinical presentation did not helped in diagnosis in most of the cases of acute knee injury and is inconclusive in cases with injuries of multiple ligaments/menisci, which corresponded with study done by Li DK et $\mathrm{al}^{5}$.

In the present study, male outnumbered female, as $80 \%$ of the patients were male and $20 \%$ were female with male to female ratio $4: 1$ with mean age for male is 31.67 years and 35.1 years for female which corresponded with the sex distribution pattern was reported in the study by Anil Madurwar et al where authors noted, out of 50 patients of knee trauma examined, 42 patients (76\%) were males and 8 of them were femalesand Singh et al where authors noted 113 men (65.31\%) and 60 women (34.69\%) out of the 173 patients with history of knee injuries. The sex distribution pattern in our study also corresponded to study by D S Shetty et al in which out of the 115 patients, $70(60.86 \%)$ were men and $45(39.14 \%)$ were women with male to female ratio of 6.4:1. The study by Jeevika $\mathrm{Mu}$ et al in 2017 found that out of 43 patients the males (84\%) are commonly affected than females (16\%).

In this study the patients with age 0 to 70 years with history of knee injury included. Different age group included i.e. 0 to 10 years, 11 to 20 years, 21 to 30 years, 31 to 40 years, 41 to 50 years, 51 to 60 years and 60-70 years. The commonest age group was 21 to 30 years in male, which comprised $38.75 \%$ of the male patients. The next common age group was 31 to 40 years with $26.25 \%$ of the male patients and $13.75 \%$ of the patients with age group 41 to 50 and 11 to 20 years. Less commonly involved age group includes 0 to 10 years and more than 60 years. The mean age was 31.675 years. The commonest age group was 21 to 30 years in female which comprised $35 \%$ of the female patients. The next common age group was 31 to 40 years with $30 \%$ of the female patients $15 \%$ for the age of 41 to 50 years and $10 \%$ for the age group 11 to 20 years. The mean age was 35.1 years for female. The age distribution pattern observed in the present study was comparable to the study of D S Shetty et al in which commonest age group was 21 to 30 years for both male and female. Singh et al also found that majority of the patients with knee injury were in third decade.

The commonest finding on MRI scanning was anterior cruciate ligament tear (partial and complete) and was present in $67 \%$ of the patients in our study which corresponded to study by Anil Madurwar et al in which authors noted $36(76 \%)$ ACL tears in total 50 knees examined on MRI. Midsubstance tear is commonest accounting $47.4 \%$ followed 
by femoral attachment $42.10 \%$ and tibial attachment $10.5 \%$. Low grade partial ACL tear was observed in $47.4 \%$ of patients and high grade partial ACL tear in $21 \%$ of patients with ACL injuries. Furthermore grade I sprain was observed in 6.6\% ACL injuries. Similar ACL injury pattern observed in the present study was comparable to the study of D S Shetty et al in which commonest knee injury is ACL injury with midsubstance tear is the commonest location of ACL tear. Singh et al also found that major knee injury is ACL tear and the commonest complete ACL tear is midsubstance tear.

Out of total 100 patients, percentage of PCL injuries detected was $15 \%$. Out of total 15 PCL injuries, partial and complete tear were noted in 6 and 6 patients respectively, total 12 PCL tears detected. Grade I sprain comprised 3 patients. In complete PCL tears, midsubstance and avulsion tear are commonest (33.33\%) followed by femoral and tibial end (16.67\%) which corresponded to study done by Anil Madurwar et $\mathrm{al}^{6}$, D J Singh et $\mathrm{al}^{8}$ and Shetty et $\mathrm{al}^{9}$ where authors noted 6\%, 5.0\% and 4.4\% PCL tear respectively. Out of total 100 patients, Grade III signal was most common in both menisci followed by grade II and grade I which corresponded to study done by Jeevika $\mathrm{Mu}$ et $\mathrm{al}^{7}$ and D S Shetty et $\mathrm{al}^{9}$. Among menisci, medial meniscus is commonly injured (38\%), followed by lateral meniscus (26\%). Posterior horn of medial meniscus was commonly injured structure among menisci noted in 30 cases against 64 meniscal tears. Next commonly injured structured is posterior horn of lateral meniscus (16.7\%) followed by anterior horn of lateral meniscus (14.1\%), body of medial meniscus (12.8\%), body of lateral meniscus (7.7\%) and anterior horn of medial meniscus (2.5\%). Some of the tears located in one part were noted extending to other parts of meniscus. Horizontal tear was commonest (12\%) followed by complex tear (11\%), bucket handle tear (07\%), radial tear (3\%) and longitudinal tear (2\%) in medial meniscus. Commonest type of tear in lateral meniscus was horizontal tear (42.3\%) followed by complex tear (26.9\%), longitudinal tear (15.5\%), vertical radial tear (11.5\%) and oblique tear (3.8\%). Similar meniscal injury pattern observed in the present study was comparable to the study of D S Shetty et al in which commonest meniscal injury is posterior horn of medial meniscus. Singh et al also found that commonest meniscal injury is posterior horn of medial meniscus. Jeevika $\mathrm{Mu}$ et al also found that among meniscal injuries medial meniscal tears (46.5\%) were more common than lateral meniscus (37.2\%) and the posterior horn of medial meniscus $(55 \%)$ is commonest site of involvement.

Out of total 100 patients complete MCL tear is noted in 1 patient, partial tear in 3 patients and grade I sprain noted in 1 patient. Similarly partial LCL tear noted in 3 patients and grade I sprain in 3 patients. It was found that MCL injury is associated with ACL and medial meniscus injuries. Out of $9 \mathrm{MCL}$ injuries, $\mathrm{ACL}$ and medial meniscus injuries were noted in 6 and 2 patients respectively. "O Donoghue's triad" was noted in 1 patient. 3 lateral meniscal tears are noted in total 12 partial LCL tear, LCL tear showed a relationship with lateral meniscus tear $(25 \%)$ which corresponded to the study done by Anil Madurwar et al.

Complete medial patellar retinaculum tear was noted in
1 patient, partial tear in 4 patients and grade I sprain in 1 patient. Patellar dislocation with medial patellar retinaculum sprain was noted in 1 patient. Patellar dislocation was associated with medial patellar retinaculum sprain which corresponded to the study done by Charles et $\mathrm{al}^{10}$. Partial tear of lateral patellar retinaculum was noted in 1 patient and grade I sprain in 1 patient.

Out of 100 patients with knee injury, bone contusions are noted in 46 patients which corresponded to the study done by Anil Madurwar et al where author noted $42 \%$ bone contusion in their study. $42.1 \%, 47.36 \%, 36.84 \%$ and $37.5 \%$ of ACL, PCL, medial meniscus and lateral meniscus injuries were associated with bone contusions. Injuries of PCL is noted commonly associated with bone contusions which corresponds to study done by Scott D Mair et a ${ }^{11}$. where author found posterior cruciate ligament injuries mostly occur with osseous and some degree of associated ligamentous injury.

Knee joint MRI improved the diagnosis of ligament and meniscal tears, muscles and tendons injuries. Bone contusions, osteochondral injuries, traumatic chondromalacic changes, joint effusion and haemarthrosis are also diagnosed well on MRI. MRI provides important clinical information that guides patient management and treatment planning.

Overall the present study showed that, MRI of the knee joint has wide approach for the diagnosis of traumatic knee injuries. This makes it as a practical, well accepted and accurate non invasive imaging technique in patients presenting with injury to knee joint and is the modality of choice in clinically suspected cases of soft tissue injury where plain radiographs are normal. Furthermore, excellent soft tissue and multiplanar planes imaging provides optimal assessment of ligaments, menisci, muscles, tendons, fibrous cartilage, joint capsule, fat bursae and bone marrow. The results of our study support the use of early MRI in case of knee injuries to guide further surgical management.

\section{CONCLUSION}

Magnetic resonance imaging of the knee is the excellent non invasive investigation tool for knee injury due to excellent contrast resolution and multiplanar imaging capabilities which provides the most detailed evaluation in cases of various soft tissue injuries of knee joint. Clinical features may suggest the soft tissue injury, but for further evaluation, MRI is necessary. MRI is unique in its ability to evaluate the ligaments, menisci, articular cartilage, articular capsule and bone marrow. Even though anatomical variants and technical artefacts can mimic a tear on MRI, it is still considered as the primary imaging tool for optimal depiction of internal derangement of knee injury. Commonest injuries detected in our study are- anterior cruciate ligament tear, tear of posterior horn of medial meniscus, bone contusions and joint effusions.

\section{REFERENCES}

1. Sanches Vaz CE, Pires De Camargo O,José De Santana $\mathrm{P}$, Valezi AC. Accuracy of magnetic resonance in identifying traumatic intraarticular knee lesions. Clinics. 2005;60(6):445-50. 
2. Kijowski R, Roemer F, Englund M, Tiderius CJ, Swärd $\mathrm{P}$, Frobell RB. Imaging following acute knee trauma. Vol. 22, Osteoarthritis and Cartilage. 2014. p. 1429-43.

3. Gupta K, Sandhu P, Galhotra R, Guleria M. Correlation of clinical, MRI and arthroscopic findings in diagnosing meniscus and ligament injuries at knee joint: A prospective study. J Orthop Allied Sci [Internet]. 2013;1(1):2.

4. Prickett WD, Ward SI, Matava MJ. Magnetic resonance imaging of the knee. Sports Med 2001;31(14):9971019.

5. Li DK, Adams ME, McConkey JP. Magnetic resonance imaging of the ligaments and menisci of the knee. Radiologic clinics of North America. 1986;24(2):20927.

6. Mu J, Reddy DLVB. Role of Mri in Meniscal Tears dr. IOSR J Dent Med Sci 2017;16(3):111-7.

7. Scientist V, Road HM. International Journal of Research and Review. 2017;4(6):50-2.

8. J.P. S, L. G, R. S, V. S, V. G. MR imaging of knee with arthroscopic correlation in twisting injuries. Indian J Radiol Imaging. 2004;14(1):33-40.

9. Shetty, Lakhkar B, Krishna G. Magnetic resonance imaging in pathologic 138 conditions of knee. Indian J Radiol Imaging. 2002;12(3):375.

10. Matava M. Patellar Tendon Ruptures. J Am Acad Orthop Surg 1996;4(6):287-96.

11. Scott D. Mair, MD, Theodore F. Schlegel, MD, Thomas J. Gill, MD in 2004, Incidence and Location of bone Bruises after Acute Posterior Cruciate Ligament Injury.

Source of Support: Nil; Conflict of Interest: None

Submitted: 11-07-2018; Accepted: 12-08-2018; Published online: 21-08-2018 